\title{
Treatment of Chronic Spontaneous Urticaria With Benralizumab: Report of Primary Endpoint Completer Analysis, Secondary and Exploratory Endpoints
}

\author{
Umesh Singh $^{1}$, Jonathan Bernstein ${ }^{2}$, Marepalli Rao ${ }^{1}$, Karen Berendts ${ }^{3}$, Xiang Zhang ${ }^{1}$, and \\ Diya Mutasim ${ }^{1}$ \\ ${ }^{1}$ University of Cincinnati College of Medicine \\ ${ }^{2}$ University of Cincinnati \\ ${ }^{3}$ Bernstein Clinical Research Center
}

October 9, 2020

\begin{abstract}
Background. Standard of care for chronic spontaneous urticaria (CSU) includes second-generation H1-antihistamines (SGAH) but is often ineffective even with four-times the FDA-recommended dose. Urticarial lesions are commonly characterized by increased lymphocytes with perivascular eosinophilic infiltrates implying a role for the interleukin-5 (IL5). Objective. This study investigated the effects of benralizumab, an anti-IL5-receptor-alpha monoclonal antibody, in human subjects with SGAHunresponsive CSU that completed all study visits. Methods. A repeated-measures, 24-week study was conducted at an urticaria specialist clinic where SGAH-unresponsive CSU patients (3 males and 9 females; age range, 32-65 years) having a median weekly Urticaria Activity Score (UAS7) of 4 and pruritus severity [?]2 were enrolled. After a baseline run-in period, subjects were treated with a subcutaneous placebo dose followed by benralizumab 30mg subcutaneously every month $(\times 3$ doses $)$ followed by two off-medication monthly-visits. The primary and exploratory endpoints were the change from baseline in UAS7 and Chronic Urticaria Quality-of-Life Score (CU-QoLTS) respectively. Secondary endpoints included peripheral blood eosinophils (eos\%) and basophils, skin eosinophilia, and differentially expressed genes (DEGs) in blood before- and after benralizumab. Results. UAS7 and CU-QoLTS significantly improved post-benralizumab compared to baseline scores in 7 of 9 subjects completing the study. Clinical improvements correlated with reduction in eos\% and inflammatory cell infiltrates in skin lesions. Biologic pathways, regulated by DEGs, involved IL-5R activity, tryptophan metabolism and Siglec-8 expression. Conclusion. Benralizumab was clinically efficacious in the treatment of subjects with SGAH-refractory CSU which correlated with several DEGs in blood. This study supports the use of benralizumab for CSU treatment.
\end{abstract}

\section{INTRODUCTION}

Chronic spontaneous urticaria (CSU) manifests as wheals \pm angioedema for $>6$ weeks of unknown cause. ${ }^{1-3}$ It affects about $1 \%$ of US adults and is associated with significant morbidity and health burden. ${ }^{4,5}$ The pathogenic mechanism of CSU is not completely understood. Thus, the first-line treatment of this condition has mostly been palliative that includes second generation H1-antihistamines (SGAH) up to 2-4 times the FDA's recommended dose. ${ }^{6}$ However, only $40 \%$ of CSU patients respond to this treatment. ${ }^{4}$ Recently, omalizumab has emerged as the preferred add-on biologic treatment for CSU patients unresponsive to SGAH. ${ }^{7,8}$ Although, omalizumab has advanced the management of CSU, there are still patients with incomplete to no response to this biologic. Moreover, for most patients who require prolonged treatment with omalizu- 
mab, its therapeutic benefit does not persist after discontinuation. These limitations emphasize the need for additional investigation of novel CSU therapies. ${ }^{4,7-9}$

Several autoimmune mechanisms have been proposed including the production of IgG antibody to FcER1 $\alpha$ subunit and anti-IgE antibodies. ${ }^{10-12}$ The roles of mast cells and eosinophils as key factors in CSU pathogenesis have also been previously investigated, ${ }^{13,14}$ which may extend beyond stimulation of the FcER1 alpha subunit on mast cells. ${ }^{15}$ Exact triggers for mast cell activation and degranulation are unknown but both receptor- and non-receptor mediated events have been suggested. ${ }^{16}$ Evidence supporting a role for the IL5 in the pathomechanism(s) of CSU is suggested by perivascular lymphocytes with eosinophilic infiltrates and an abundant IL5 in lesional vs. non-lesional skin of CSU patients. ${ }^{17}$ Benralizumab, a humanized anti-IL5receptor- $\alpha$ monoclonal antibody, has been demonstrated to be clinically effective by decreasing eosinophil counts in blood, induced sputum, and lung tissue in persistent eosinophilic asthma. ${ }^{18-20}$ However, the efficacy of benralizumab at controlling CSU has never been studied. It was thus hypothesized, that benralizumab would be efficacious in SGAH refractory CSU as measured by Urticaria Activity Score over 7 days (UAS7) as a primary objective supported by the major transcriptomic changes as a secondary objective, and the Chronic Urticaria Quality of Life Questionnaire total score (CU-QoL TS) as an exploratory objective. ${ }^{21-23}$ We have recently reported the intent-to-treat primary endpoint data analysis. ${ }^{24}$ Here we now report the primary, secondary and exploratory endpoints of subjects completing all study visits.

\section{METHODS}

\section{Study Design, Outcomes And Sample Size}

This single-blinded, one-arm, repeated-measures, single-center study was conducted at a specialist urticaria clinic (Figure 1). The inclusion criteria, mentioned in S-Table 1, were SGAH-unresponsive adult CSU subjects with a weekly urticaria activity score (UAS7) of [?]4 and pruritus score of [?]2. The primary endpoint was the percentage of CSU subjects with a significant clinical response to 3 doses of benralizumab $30 \mathrm{mg}$ versus placebo. Clinical response was measured by UAS7 (range 0-42), a validated questionnaire that quantifies wheal and pruritus severity over seven days week each recorded on a $0-3$ scale. ${ }^{21}$ For this study, a significant clinical response was defined as a minimally detectable change (MDC) in UAS7 of 14 from the baseline score. For an MDC of 14 with a minimum sample size of 6 CSU subjects, the power of the study was calculated to be $>0.9$ (alpha=0.05). All urticaria medications were discontinued during the study period except rescue medications. A medication history for use of SGAHs and other urticarial therapies including biologics were obtained from all subjects at their baseline visit.

Secondary endpoints included the percent reduction in inflammatory cell counts in peripheral blood and skin, and the differential gene expression using RNA-sequencing in peripheral blood pre- and post-treatment with benralizumab. An objective response to benralizumab was determined by measuring differences of inflammatory cell infiltrates (lymphocytes, basophils) in skin biopsies at visit 2 (post-placebo/pre-benralizumab) vs. visit 5 (4 weeks post-benralizumab), and relative white cell counts in peripheral blood sampled at baseline and at each post-treatment visit. An exploratory endpoint was the validated chronic urticaria quality of life questionnaire (CU-QoL summarized in S-Table 2). ${ }^{25,26}$

\section{Intervention}

CSU subjects were treated with a single subcutaneous dose of placebo at visit 1 , followed by three subcutaneous doses of benralizumab $30 \mathrm{mg}$ at 4 -week intervals (visits 2, 3, and 4). The non-CSU subjects, recruited for comparison blood and skin biopsy control samples, received no treatment.

\section{Assessment Methods}


UAS7 and CU-QoL Total Score: At each visit, the subjects provided their UAS7 and CU-QoL total scores for the previous 4 weeks.

Blood Sampling: Blood samples were collected prior to any treatment intervention at all visits in EDTA tubes for total and differential counts and at visits 2 (post-placebo) and 5 (4-weeks after $3^{\text {rd }}$ dose of benralizumab) in PAXgene Blood RNA (Qiagen) tubes for RNA-sequencing.

Total RNA Extraction and RNA-Seq For Differential Gene Expression Profiling from Whole Blood:Directional RNA-seq was performed by the Genomics, Epigenomics and Sequencing Core at the University of Cincinnati. ${ }^{27,28}$ RNA extraction and sequencing methods are described in the supplement.

Skin Biopsy Collection, Processing, and Analysis : Two full-thickness skin biopsies were taken using Acu-Punch Kit Ultra (4mm Acu-Punch, Acuderm) from lesional (active hives) and nearby non-lesional (normal) skin from CSU subjects at visit 2 and at visit 5 for histologic and transriptomic analysis. Lesional biopsies (if present in non-responders or partial responders) and non-lesional biopsies at visit 5 were performed at locations close to the baseline samples. For complete benralizumab responders (i.e., subjects with UAS7=0 at visit 5), only non-lesional skin was biopsied. Normal skin biopsies were collected once from non-CSU control subjects using identical methods. Biopsies for histopathological analysis were transported prior to processing in formalin and for RNA-sequencing in RNAlater tubes. Skin biopsy transcriptomic analyses will be presented in a subsequent manuscript.

\section{STATISTICAL ANALYSIS}

Demographic Analysis : The mean age of CSU subjects by gender and race, and the duration of disease between responders vs. non-responders were compared using t-tests.

\section{Primary and Exploratory Endpoint (Clinical Efficacy) Analysis}

It was assumed that hives were not self-limiting and any improvement in outcomes during the study were because of interventions. For primary end-point analysis (i.e., UAS7), data from subjects completing the study $(\mathrm{n}=9)$ were analyzed; whereas for the CU-QoL total score exploratory end-point, an intent-to-treat $(\mathrm{n}=12)$ and complete responder $(\mathrm{n}=9)$ analysis was performed. A repeated-measures mixed-model regression analysis using the 'glimmix' procedure was used to fit a generalized-linear-mixed model for comparison of UAS7 and CU-QoL total scores between visits and between responder phenotypes adjusted for their baseline severity of disease. Correlational analysis between UAS7 and CU-QoL total scores were performed to associate clinical improvements with improved QoL.

\section{Secondary Endpoint Analysis}

Cell Infiltrates in Lesional and Non-Lesional Skin Biopsies.Quantitative analysis of mononuclear and mast cells observed around postcapillary venules in lesional skin vs. non-lesional skin at visits 2 and 5 were performed to determine the effect of benralizumab on cell infiltration as its possible therapeutic mechanism in CSU.

Peripheral Blood White Cell Counts. Relative white cell counts in peripheral blood (eos\%, basophil\%) over the entire duration of the study was performed to determine any significant associations of changes in eos\% or basophil\%, due to treatment with benralizumab, with the corresponding mean $\Delta \mathrm{UAS} 7$ and differential gene expression in peripheral blood pre- and post-benralizumab.

Comparison Between Responders And Non-Responders To Benralizumab. Analysis of the effect of benralizumab on the differential white cell count in peripheral blood between responders and non-responders was performed to determine effect.

Differential Gene Expression In Blood. For differential gene expression in peripheral blood, the RNA sequence reads were aligned to the reference genome using the TopHat aligner ${ }^{29}$, and reads aligning to each known transcript were counted using Bioconductor packages for next-generation sequencing data analysis. ${ }^{30}$ 
DEGs were analyzed in Ingenuity Pathway Analysis (IPA) platform (Qiagen) for determination of pathway enrichment and upstream regulators.

\section{RESULTS}

Subject Characteristics. Twelve CSU and 6 non-CSU (control) subjects were enrolled. The mean age of CSU subjects (F:M-9:3, Black:White-2:10) was 47.3 years. The average duration (with $95 \%$ CI) of CSU symptoms was $7.0(0.2-18)$ years. Most CSU subjects were prescribed diphenhydramine or hydroxyzine as rescue therapy. Nine subjects completed the study. Three (25\%) of the 12 subjects dropped out of the study after the first benralizumab dose (one due to personal issues, one lost to follow-up and one due to nonresponse); among them, two showed a trend to response and one was a non-responder who had previously also not responded to omalizumab.

\section{Primary and Exploratory Outcomes}

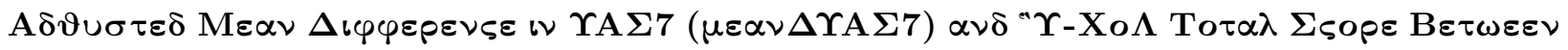

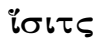

Table 1 and Figure 2 summarize the mean differences in UAS7 and CU-QoL between visits adjusted for the severity and the duration of disease at enrollment. The baseline UAS7 ranged from 16 to 42 between subjects, implying baseline variations in disease severity from moderate to severe CSU. The primary outcome, (i.e. the mean $\triangle \mathrm{UAS} 7$ ), decreased significantly 4 -weeks after the $3 \mathrm{rd}$ benralizumab dose (visit 5 ) versus postplacebo (visit 2) and baseline (visit 1). Five subjects had a UAS7=0 and two had a UAS [?]6 at visit 5 or 6 . The effect of benralizumab on the UAS7 was statistically and clinically significant based on the sustained mean differences between follow-up Visit 6 (i.e., 8 weeks after the last benralizumab dose) vs. the post-placebo Visit $2(\Delta \mathrm{UAS} 7=15.2[3.2,27.2], \mathrm{p}=0.006)$ or vs. the baseline Visit 1 ( $\Delta \mathrm{UAS} 7=17.7[5.3$, $30.1], \mathrm{p}=0.001$ ). Both components of the UAS7 (i.e., pruritus severity and wheal size) improved to a similar extent. The differences in CU-QoL total scores were statistically significant at several time points mostly for the pruritus and wheals components. UAS7 and CU-QoL total scores had significant positive correlation $(\rho$ $=0.9, \mathrm{p}<0.0001)$. No drug-related adverse events were reported throughout the study.

\section{Regulation of Peripheral Blood Count by Benralizumab}

Treatment with benralizumab in CSU decreased eos\% significantly. The averages (min., max.) of eos\% at baseline, post-placebo visit, and 4-weeks after the third benralizumab dose were $3.8(0,10), 3(0.2,6.3)$, and $0(0,0.2)$, respectively. Significant differences were observed immediately after the first dose of benralizumab (visit 3) compared to baseline (visit 1) (mean difference=3.7, $\mathrm{p}=0.0002$ ) (Table 2 \& S-Figure 1 ). These differences persisted 8-weeks after the last benralizumab dose (Visit 6) and were seen for responders as well as non-responders.

\section{Regulation of Cutaneous Inflammatory Cell Infiltrates by Benralizumab}

At baseline, the number of mononuclear cells (predominantly lymphocytes) around perivascular capillaries in the lesional skin were significantly higher than in the adjacent non-lesional skin (mean difference $=18 \pm 5$ cells/field, p=0.006) (Figure $\mathbf{3} \mathbf{B}, \mathbf{D}, \mathbf{F}$ ). Such differences were observed qualitatively when compared with samples from non-CSU control subjects (Figure 3 A ). Five of 9 subjects completing the study (56\%) who responded completely to benralizumab had reduced mononuclear cell infiltrates. Comparison of mononuclear cell infiltrates from the two partial and two non-responder CSU subjects post-benralizumab vs. post-placebo, also was suggestive of benralizumab's effect (Figure $\mathbf{3} \mathbf{B}, \mathbf{E}, \mathbf{F}$ ) with the mean reduction being approximately 17 cells/field $(\mathrm{p}=0.07)$. Skin infiltrates from non-lesional skin post-benralizumab were qualitatively similar to non-CSU (control) skin samples (Figure 3 A \& C ). At baseline, the average mast cell count in lesional skin was higher compared to adjacent non-lesional skin, (average difference $=9$ cells/field, $\mathrm{p}=0.2$ ). These differences persisted after treatment with benralizumab (S-Figure 2 ).

\section{Differences Between Responders And Non-Responders to Benralizumab}


The response to benralizumab was defined, based on a similar definition using UAS7 in a previous study, as "no response" if at any time during the 16 weeks after the first benralizumab injection there was $<40 \%$ improvement in UAS7, and as "response" if the UAS7 was [?]6. ${ }^{31}$ Seven (78\%) of 9 subjects completing the study were thus classified as responders; among them five were complete responders (i.e., no hives at visits 5 or 6$)$. Between responders and non-responders, their age difference (51.6 vs. $53, \mathrm{p}=0.9)$ and the duration of disease ( 5 vs. 9 years, $\mathrm{p}=0.5$ ) were not statistically significant. The adjusted mean UAS7 percentage difference from Visit 1 till Visit 5 was $-84 \%$ for responders and $+7.5 \%$ for non-responders, $\mathrm{p}=0.0009$ (STable 3). These differences were adjusted for the length of disease, subject age, and peripheral blood eosinophil percentages (eos\%). Although there were no significant differences for UAS7 at baseline between responders versus non-responders $(\Delta \mathrm{UAS} 7=-3.4, \mathrm{p}=0.9)$, there were significant differences observed at visit $5(\triangle \mathrm{UAS} 7=-30.4, \mathrm{p}=0.03)$. The average length of disease between responders and non-responders was 5.1 vs. 9.0 years, $\mathrm{p}=0.06$. Interestingly, there were significant differences between responders and non-responders at baseline (visit 1) for peripheral blood eos\% $(-6 \pm 1, \mathrm{p}=0.001)$ and basophil\% $(-1.2 \pm 0.2, \mathrm{p}=0.02)$, which were not observed at visit 5 (eos\%: 0 $01, \mathrm{p}=0.9$; basophil\%: $0.2 \pm 0.2, \mathrm{p}=0.8)$ (S-Table 3 ).

\section{Secondary Outcome: Differential Gene Expression in Blood Pre- Vs. Post-Benralizumab}

Relevant blood DEGs between visit 2 (post-placebo) and visit 5 (post-benralizumab) samples include IDO1, PIK3R6, SIGLEC8, CYP4F12, IL5RA, ALOX15, and IL1R1 (S-Table 4). These DEGs imply regulation by benralizumab of tryptophan metabolism, eosinophilic apoptosis, mast-cell mediator release and cytokinesignaling mediated by IL5R and IL1R1. Normalized expression levels of several of these DEGs correlated significantly with UAS7, eos\% and CU-QoL Total Scores (S-Table 5 ). Additionally, several of these blood DEGs such as IDO1, PTGDR2, ALOX15, IL1RL1, and SLC29A1 may represent biomarkers that can distinguish complete responders from non-responders and partial responders. Interestingly, the predicted upstream regulator for all these genes, as determined by IPA, is IL4 ${ }^{32,33}$ suggesting that IL5-R antagonism may work through common pathways that also regulate IL4 activity such as through Nuclear Factor of Activated T-cells (NFAT).

\section{Discussion}

This study demonstrated that benralizumab resulted in significant clinical improvement in subjective and objective measures in $78 \%$ (7/9) of CSU patients unresponsive to H1-antihistamines who completed the study, which was sustained for two months after the last treatment dose. Furthermore, an overall difference of UAS7 greater than the MDC value of 14.7 between baseline and end of study post-benralizumab treatment implies that this finding is also clinically meaningful (Table 1). These results are consistent with the intentto-treat primary endpoint analysis recently reported ${ }^{24}$. Comparison of benralizumab responders $(\mathrm{n}=7)$ to non-responders $(\mathrm{n}=2)$ revealed that the average baseline UAS7 among treatment responders was less than non-responders, although this difference was not statistically significant ( 27 vs. $37.5, \mathrm{p}=0.5$ ). This suggests that clinical improvement among responders was independent of baseline disease severity. These observations support the use of benralizumab for treatment of SGAH unresponsive CSU and provide evidence for a pathogenic role for infiltrating eosinophils. Although significant differences were observed between responders and non-responders at baseline for relative blood eosinophil $(2.2 \%$ vs. $7.9 \%, \mathrm{p}=0.001)$ and basophil counts $(0.3 \%$ vs, $1.5 \%, \mathrm{p}=0.01)$, these differences were minimal and no longer apparent post-treatment (eos\%: 0.0 vs. $0.0, \mathrm{p}=0.99$; basophil $\%$ : 0.4 vs. $0.2, \mathrm{p}=0.88$ ). Thus, non-responsiveness to benralizumab as measured by changes in UAS7 are likely due to other mechanistic factors unrelated to the presence of eosinophils which are eliminated by blocking IL-5R. Further investigation is required to determine the relationship between baseline peripheral blood eosinophil levels and benralizumab's response in CSU.

Not surprisingly, as seen in previous asthma studies, the effect of benralizumab on eos\% was observed immediately after the first dose and was sustained for 8 -weeks after the last dose. ${ }^{34}$ The more prolonged effect of benralizumab in the complete responders after discontinuation, compared to what has been reported after discontinuation of omalizumab in controlled CSU patients, may be explained by modification of biologic pathways important for promoting persistent cutaneous inflammation associated with CSU. Additional objective evidence to support this conclusion is the observed effect of benralizumab in decreasing the numbers 
of lymphocytes observed in non-lesional skin of complete responders compared to baseline lesional biopsies. Eosinophils were also significantly reduced in the lesional skin of non-responder and partial responder CSU subjects $(\mathrm{n}=4)$ 4-weeks after the last dose of benralizumab compared to baseline lesional skin biopsies, as would be expected given benralizumab's primary mechanism of action (Figure 3 D-E ).

A decrease in UAS7 should reflect an improved CSU-related QoL since both outcomes measure wheals and pruritus, the two main clinical features of CSU. As expected, the CU-QoL and UAS7 post-treatment values were significantly correlated $\left(\mathrm{r}^{2}=0.9, \mathrm{p}<0.0001\right)$, which support the internal consistency of our data. Besides the pruritus and wheal component, the most significant improvement in CU-QoL were reported for urticarial interference with physical activities, sleep and spare time (S-Table 6) .

Improvements in UAS7 and CU-QoL for this pilot study are comparable to the efficacy of omalizumab in CSU, as determined in a previous pilot study (S-Table $\mathbf{7}$ ), with nearly the same percentage of complete responders to benralizumab as omalizumab. ${ }^{35}$ It is important to note, that when the larger Phase 3 omalizumab study (ASTERIA I trial) for the treatment of CSU was conducted based on the results of the earlier feasibility study, the number of patients with $50 \%$ reduction in mean UAS7 from baseline revealed a $35.8 \%$ complete response and a $51.9 \%$ partial response. ${ }^{36}$ This demonstrates that the omalizumab pilot study was a good indicator of expected outcomes in the larger multicenter clinical trial.

Given benralizumab's mechanism of action as an IL-5R antagonist, its effect in the treatment of CSU is not outwardly apparent. However, DEGs in blood before and after benralizumab has provided greater insight into some biologic pathways that may explain its efficacy in the treatment of CSU (S-Table 4 and 5 ). Urticarial wheals are clinical manifestations of altered vasoactive and cellular infiltration kinetics resulting from inappropriate activation of dermal mast cells in up to $50 \%$ of cases by $\operatorname{IgG}$ autoantibodies directly targeting the high affinity IgE mast cell receptor ( FcER1-alpha subunit) or IgE affixed to these receptors. ${ }^{37}$ The role of possible autoimmune mechanisms in CSU, although not directly investigated in this study, could be explained by our blood transcriptomic data which demonstrated that benralizumab downregulated Indoleamine-2,3-Dioxygenase (IDO) pathway expression. IDO is an interferon- $\gamma$-inducible enzyme that catalyzes tryptophan degradation and drives Th2-mediated inflammation. ${ }^{38}$ Elevated tryptophan degradation has been shown to correlate with disease activity previously reported in other autoimmune rheumatological conditions. ${ }^{39,40}$ Thus, benralizumab-mediated inhibition of IDO activity may improve CSU by restoring the basal tryptophan metabolic state from a hypercatabolic state leading to immune homeostasis ${ }^{41}$ consistent $^{2}$ with what other studies have reported. ${ }^{42}$

A minimal persistent inflammation in CSU is maintained by infiltrating inflammatory cells that release proinflammatory mediators, cytokines/chemokines and adhesion molecules capable of recruiting and activating other effector cell types. ${ }^{14}$ The sustained clinical improvement by benralizumab in $5 / 9$ CSU subjects until at least 4 -weeks after the last dose, versus baseline, is reflected by a significant reduction of peripheral blood inflammatory cell counts, which was also seen in partial- and non-responders $(\mathrm{n}=4)$ (Figure 3 ). This is supported by benralizumab's downregulation of DEGs in blood transcriptomes that control cytokine/chemokine signaling pathways (S-Table 4 ) and SIGLEC-8 gene expression. Since SIGLEC-8 is expressed on human eosinophils and mast cells, this finding further supports a role for IL5-receptor-mediated signaling of inflammatory responses involving mast cells and eosinophils. ${ }^{43}$

The study helps to better understand potential alternative medications in patients not responding to high dose SGAH. The number of complete responders was remarkably high. The blood DEGs that were significantly associated with response to benralizumab were IDO1, PTGDR2, ALOX15, IL1RL1, and SLC29A1 all of which are regulated upstream by IL4. In this context, it is worth mentioning that previous studies have reported increased serum concentrations of IL4 during the appearance of urticarial lesions in CSU patients. ${ }_{44}$ Among those DEGs, the expression of prostaglandin D2 receptor (PTGDR2) was most evidently related to the response status (i.e., complete, partial or no-response to benralizumab). Complete responders had under-expression of PTGDR2, indicated by a negative z-score (z-score $=-0.65)$; but, partial or no-responders had persistent over-expression of PTGDR2, indicated by positive z-scores, even after treatment with benralizumab. The expression levels of this gene were correlated with UAS7, and also with absolute and relative 
eosinophil or basophil scores. Previous studies have reported increased prostaglandin D2 in venous blood draining urticarial wheals. ${ }^{16}$ In this context, over-expression of PTGDR2 might perpetuate or exaggerate the inflammatory condition that may be unresponsive or less responsive to monotherapy with an IL-5 antagonist alone. Fevipiprant (NVP-QAW039, Novartis), a potent and selective PTGDR2 antagonist which has previously been assessed for safety and tolerability in human studies ${ }^{45}$ could be studied in partial or non-responsive benralizumab treated patients to confirm the relevance of this genetic biomarker.

\section{Limitations}

This analysis considered only a limited number of demographic and clinical factors so clinical characteristics of subjects predicting a therapeutic response to Benralizumab could not be adequately assessed. Other limitations are the small number of subjects enrolled and the single blind pilot study design. Although this was a small study, there was an adequate power to predict a clinical significance in the UAS7 primary endpoint. With respect to the study design, it should be emphasized that pilot studies represent a fundamental phase of the clinical research process and are essential for determining the feasibility of pursing a larger clinical trial ${ }^{50}$ which is supported by the results of this study.

\section{Conclusion}

Treatment of SGAH-unresponsive CSU subjects, with benralizumab 30mg subcutaneously monthly for 3 successive months, versus a placebo dose, significantly improved disease severity and QoL as measured by UAS7 and CU-QoL total score, respectively. These observations support the use of benralizumab for treatment of SGAH unresponsive CSU and provide evidence for a pathogenic role for infiltrating eosinophils. These data provide justification for a double-blind, placebo-controlled multicenter study in a large patient population currently underway.

\section{TABLES}

Table 1. Complete responder analysis $(n=9)$ for UAS7 Primary Endpoint and the exploratory CU-QoL endpoint.

\begin{tabular}{llll}
\hline Comparison Timepoints & Mean-difference $( \pm$ confidence limits) in UAS7 between timepoints & Adj P & Mean-difference \\
\hline Visit 0 vs. Visit 1 & $3.3(-5.6,14.4)$ & 0.82 & $3.9(-6.4,14.3)$ \\
Visit 0 vs. Visit 2 & $3.3(-6.7,13.3)$ & 0.94 & $3.5(-6.9,13.9)$ \\
Visit 0 vs. Visit 3 & $11.3(0.3,22.3)$ & 0.04 & $3.2(-7.1,13.6)$ \\
Visit 0 vs. Visit 4 & $11.1(0.1,22.0)$ & 0.05 & $8.4(-2.8,19.7)$ \\
Visit 0 vs. Visit 5 & $17.6(6.6,28.6)$ & $<0.0001$ & $15.1(3.9,26.3)$ \\
Visit 0 vs. Visit 6 & $19.4(8.3,30.4)$ & $<0.0001$ & $12.3(1.1,23.5)$ \\
Visit 1 vs. Visit 5 & $13.2(2.5,23.9)$ & 0.006 & $11.2(0.4,21.9)$ \\
Visit 1 vs. Visit 6 & $15.0(4.2,25.7)$ & 0.0006 & $8.4(-2.4,19.1)$ \\
Visit 2 vs. Visit 5 & $14.3(3.5,25.0)$ & 0.002 & $11.6(0.8,22.4)$ \\
Visit 2 vs. Visit 6 & $16.0(5.3,26.8)$ & $<0.0001$ & $8.8(-1.9,19.6)$ \\
\hline
\end{tabular}

Between Visits Adjusted for Multiple Comparisons in a Generalized Linear Mixed Model. Analysis is based on the 9 subjects completing the trial; Data is adjusted for baseline severity (using random intercept in the mixed model linear regression analysis) and the duration of disease for each subject (Visit $\mathbf{0}=$ baseline; Visit $\mathbf{1}=$ pre-placebo treatment; Visit $\mathbf{2}=$ post-placebo/pre-benralizumab visit; Visits $\mathbf{2 - 4}=$ Benralizumab 30 mg s.c. treatment; Visit $\mathbf{5}=4$ week post-Benralizumab visit (determination of primary efficacy end-point); Visit $\mathbf{6}=8$ week post-benralizumab follow-up visit (determination of long term efficacy and adverse events); * primary and exploratory outcomes are shaded (Adj $p<.05$ ).

Table 2. Differences in Relative Eosinophil Counts (Eos\%) Between Visits in CSU Subjects Before and After Treatment with Benralizumab 


\begin{tabular}{lll}
\hline Eos\% Comparison Timepoints & Average Difference $(\mathbf{9 5 \%}$ CI) & Adj P value* \\
Visit 1 vs. Visit 3 & $3.7(1.4,6.1)$ & 0.0002 \\
Visit 1 vs. Visit 4 & $3.9(1.5,6.3)$ & 0.0002 \\
Visit 1 vs. Visit 5 & $3.8(1.4,6.2)$ & 0.0002 \\
Visit 1 vs. Visit 6 & $3.5(1.1,6.0)$ & 0.0010 \\
Visit 2 vs. Visit 3 & $2.8(0.3,5.2)$ & 0.02 \\
Visit 2 vs. Visit 4 & $2.9(0.5,5.4)$ & 0.01 \\
Visit 2 vs. Visit 5 & $2.8(0.4,5.3)$ & 0.02 \\
Visit 2 vs. Visit 6 & $2.6(0.01,5.1)$ & 0.05 \\
\hline
\end{tabular}

*P value adjusted for multiple comparisons between visits

\section{FIGURES}

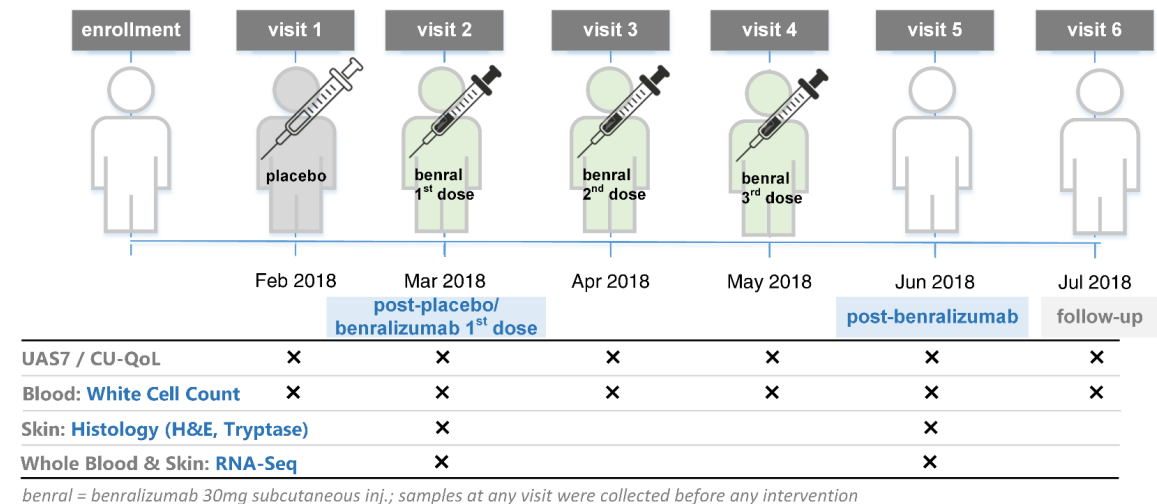

Figure 1. Study Design: After enrollment (Visit 0), a matching drug placebo was administered subcutaneously at visit 1 . Benralizumab $30 \mathrm{mg}$ was then administrated subcutaneously at three consecutive monthly intervals (visits 2, 3, and 4). Clinical outcomes (UAS7, CU-QoL) were recorded at each visit. Blood samples were collected at each visit for total and differential blood cell counts. Blood was also collected at Visits 2 and 5 for RNA-sequencing. Skin biopsies (from lesional and non-lesional skin) were obtained at visit2 (post-placebo/pre-benralizumab treatment) and again at visit 5 (4 weeks after last benralizumab treatment) from all CSU patients for histology and RNA-sequencing. Similar skin and blood samples were obtained from 5 non-CSU healthy controls at visit 0 only. Vital sign parameters were

measured at every visit. RNA-extracted from blood and skin biopsies were stored at $-80^{\circ} \mathrm{C}$ for later use. 


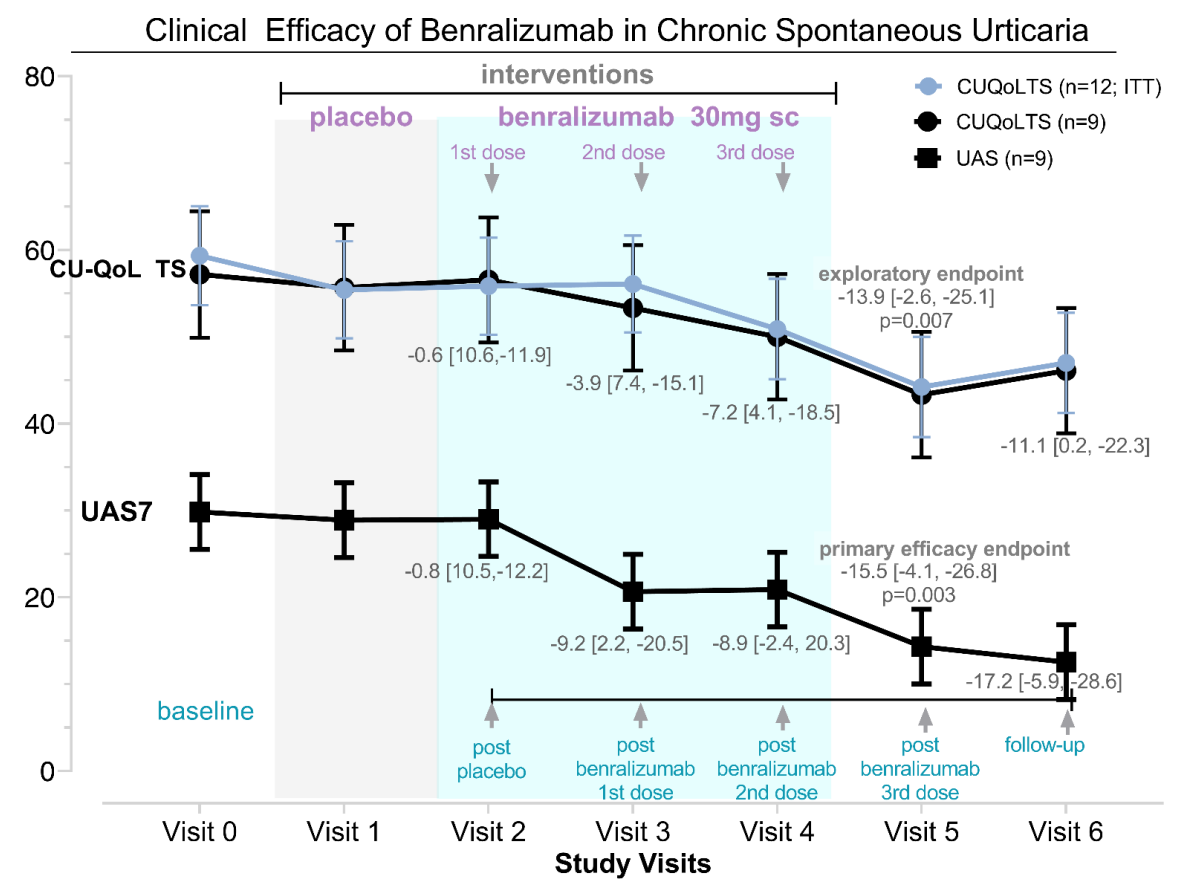

Figure 2 . Line plots illustrating UAS7 and CU-QoL Total Score at baseline and at each successive study visit. Differences in UAS7 between Visit 5 (4 weeks after last benralizumab dose) vs. Visit 0 or 1 (baseline) or vs. Visit 2 (post-placebo visit) are summarized for the 9 subjects completing the study; similar data for CU-QoL total score is shown for 9 subject completing the study and the intent to treat analysis for all 12 subjects enrolled.

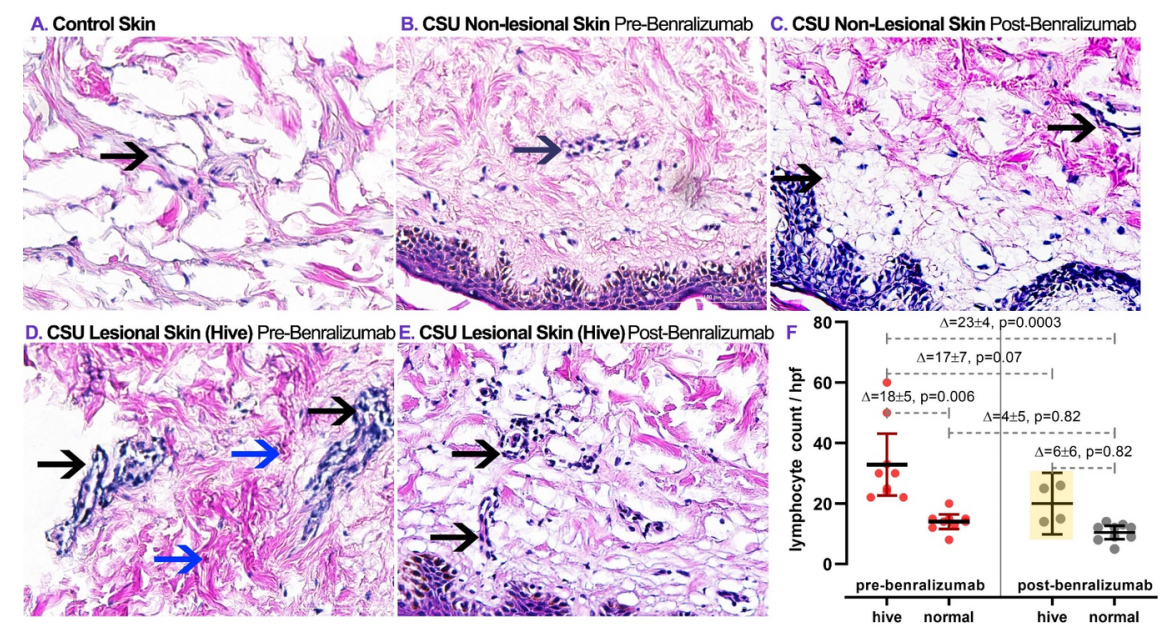

Figure 3. H\&E Stains of Skin Biopsies at Visit 2 (pre-benralizumab) and Visit 5 (post-benralizumab); purple-stained cells represent predominantly lymphocytes depicted by black arrows; red (eosinophilic)-stained cells represent eosinophils depicted by blue arrows. Panel A: Biopsy from a healthy non-CSU control subject showing scant numbers of perivascular lymphocytes and no eosinophils. Panels B and C: Non-lesional skin in a CSU complete responder showing scant numbers of perivascular lymphocytes and no eosinophils pre- and post- benralizumab treatment. Panels D: Representative histopathological section of lesional skin in a CSU complete or partial responder showing dense perivascular lymphocytes and eosinophils pre-benralizumab 
treatment (Visit 2). Panel E: Persistent but decreased perivascular lymphocytes with a complete absence of eosinophils post-benralizumab treatment (Visit 5) from hives in a partial responder. Panel F: Analysis of lymphocytic infiltrates in urticarial lesions and adjacent normal skin in all subjects completing the study $(\mathrm{n}=9)$. Pre-benralizumab samples (urticarial and normal skin) were taken from all nine subjects. Postbenralizumab urticarial samples (shaded yellow; $n=4$ ) include lesional skin samples from 2 partial responders and 2 non-responders. Post-benralizumab normal samples $(n=9)$ include samples from all complete responder subjects and adjacent normal skin from partial and non-responders).

\section{References}

1. Amin P, Bryson T, Krall D, Levin L, Eckman J, Bernstein JA. Clinical Characterization of Chronic Urticaria Phenotypes to Predict Treatment Outcomes. Journal of Allergy and Clinical Immunology. 131(2):AB27.

2. Asero R, Cugno M, Tedeschi A. Eosinophils in chronic urticaria: supporting or leading actors? The World Allergy Organization journal. 2009;2(9):213-217.

3. Vestergaard C, Deleuran M. Chronic spontaneous urticaria: latest developments in aetiology, diagnosis and therapy. Therapeutic advances in chronic disease. 2015;6(6):304-313.

4. Amin P, Levin L, Holmes SJ, Picard J, Bernstein JA. Investigation of patient-specific characteristics associated with treatment outcomes for chronic urticaria. The journal of allergy and clinical immunology In practice. 2015;3(3):400-407.

5. O'Donnell BF. Urticaria: impact on quality of life and economic cost.Immunology and allergy clinics of North America.2014;34(1):89-104.

6. Zuberbier T, Aberer W, Asero R, et al. The EAACI/GA(2)LEN/EDF/WAO guideline for the definition, classification, diagnosis and management of urticaria. Allergy. 2018;73(7):1393-1414.

7. Maurer M, Rosen K, Hsieh HJ, et al. Omalizumab for the treatment of chronic idiopathic or spontaneous urticaria. N Engl J Med.2013;368(10):924-935.

8. Saini SS, Bindslev-Jensen C, Maurer M, et al. Efficacy and safety of omalizumab in patients with chronic idiopathic/spontaneous urticaria who remain symptomatic on H1 antihistamines: a randomized, placebo-controlled study. The Journal of investigative dermatology. 2015;135(1):67-75.

9. Horiuchi T, Weller PF. Expression of vascular endothelial growth factor by human eosinophils: upregulation by granulocyte macrophage colony-stimulating factor and interleukin-5. American journal of respiratory cell and molecular biology. 1997;17(1):70-77.

10. Yu L, Buttgereit T, Stahl Skov P, et al. Immunological effects and potential mechanisms of action of autologous serum therapy in chronic spontaneous urticaria. J Eur Acad Dermatol Venereol.2019;33(9):17471754 .

11. Kolkhir P, Andre F, Church MK, Maurer M, Metz M. Potential blood biomarkers in chronic spontaneous urticaria. Clin Exp Allergy.2017;47(1):19-36.

12. Izaki S, Toyoshima S, Endo T, et al. Differentiation between control subjects and patients with chronic spontaneous urticaria based on the ability of anti-IgE autoantibodies (AAbs) to induce FcepsilonRI crosslinking, as compared to anti-FcepsilonRIalpha AAbs. Allergol Int. 2019;68(3):342-351.

13. Jain S. Pathogenesis of chronic urticaria: an overview.Dermatol Res Pract. 2014;2014:674709.

14. Caproni M, Giomi B, Volpi W, et al. Chronic idiopathic urticaria: infiltrating cells and related cytokines in autologous serum-induced wheals. Clin Immunol. 2005;114(3):284-292.

15. Asero R, Tedeschi A, Marzano AV, Cugno M. Chronic urticaria: a focus on pathogenesis. F1000Res. $2017 ; 6: 1095$. 
16. Church MK, Kolkhir P, Metz M, Maurer M. The role and relevance of mast cells in urticaria. Immunol Rev. 2018;282(1):232-247.

17. Caproni M, Volpi W, Macchia D, et al. Infiltrating cells and related cytokines in lesional skin of patients with chronic idiopathic urticaria and positive autologous serum skin test. Exp Dermatol.2003;12(5):621-628.

18. Bel EH, Ten Brinke A. New Anti-Eosinophil Drugs for Asthma and COPD: Targeting the Trait! Chest. 2017;152(6):1276-1282.

19. Khorasanizadeh M, Eskian M, Assa'ad AH, Camargo CA, Jr., Rezaei N. Efficacy and Safety of Benralizumab, a Monoclonal Antibody against IL-5Ralpha, in Uncontrolled Eosinophilic Asthma. Int Rev Immunol.2016;35(4):294-311.

20. Varricchi G, Bagnasco D, Borriello F, Heffler E, Canonica GW. Interleukin-5 pathway inhibition in the treatment of eosinophilic respiratory disorders: evidence and unmet needs. Curr Opin Allergy Clin Immunol. 2016;16(2):186-200.

21. Hawro T, Ohanyan T, Schoepke N, et al. The Urticaria Activity Score-Validity, Reliability, and Responsiveness. The journal of allergy and clinical immunology In practice. 2018;6(4):1185-1190 e1181.

22. Mathias SD, Dreskin SC, Kaplan A, Saini SS, Spector S, Rosen KE. Development of a daily diary for patients with chronic idiopathic urticaria. Ann Allergy Asthma Immunol. 2010;105(2):142-148.

23. Mathias SD, Crosby RD, Zazzali JL, Maurer M, Saini SS. Evaluating the minimally important difference of the urticaria activity score and other measures of disease activity in patients with chronic idiopathic urticaria. Ann Allergy Asthma Immunol. 2012;108(1):20-24.

24. Bernstein JA, Singh U, Rao MB, Berendts K, Zhang X, Mutasim D. Benralizumab for Chronic Spontaneous Urticaria. 2020;383(14):1389-1391.

25. Choi WS, Lim ES, Ban GY, et al. Disease-specific impairment of the quality of life in adult patients with chronic spontaneous urticaria.Korean J Intern Med. 2018;33(1):185-192.

26. Baiardini I, Pasquali M, Braido F, et al. A new tool to evaluate the impact of chronic urticaria on quality of life: chronic urticaria quality of life questionnaire (CU-QoL). Allergy.2005;60(8):1073-1078.

27. Walsh KB, Zhang X, Zhu X, et al. Intracerebral Hemorrhage Induces Inflammatory Gene Expression in Peripheral Blood: Global Transcriptional Profiling in Intracerebral Hemorrhage Patients. DNA Cell Biol.2019;38(7):660-669.

28. Sharma M, Zhang X, Huang S. Integrate Imaging Flow Cytometry and Transcriptomic Profiling to Evaluate Altered Endocytic CD1d Trafficking.J Vis Exp. 2018(140).

29. Trapnell C, Pachter L, Salzberg SL. TopHat: discovering splice junctions with RNA-Seq. Bioinformatics. 2009;25(9):1105-1111.

30. Huber W, Carey VJ, Gentleman R, et al. Orchestrating high-throughput genomic analysis with Bioconductor. Nat Meth. 2015;12(2):115-121.

31. Ohanyan T, Schoepke N, Bolukbasi B, et al. Responsiveness and minimal important difference of the urticaria control test. J Allergy Clin Immunol. 2017;140(6):1710-1713 e1711.

32. Snodgrass RG, Brune B. Regulation and Functions of 15-Lipoxygenases in Human Macrophages. Front Pharmacol. 2019;10:719.

33. Musso T, Gusella GL, Brooks A, Longo DL, Varesio L. Interleukin-4 inhibits indoleamine 2,3-dioxygenase expression in human monocytes. Blood. 1994;83(5):1408-1411.

34. Castro M, Wenzel SE, Bleecker ER, et al. Benralizumab, an anti-interleukin 5 receptor alpha monoclonal antibody, versus placebo for uncontrolled eosinophilic asthma: a phase $2 \mathrm{~b}$ randomised dose-ranging study. 
Lancet Respir Med. 2014;2(11):879-890.

35. Kaplan AP, Joseph K, Maykut RJ, Geba GP, Zeldin RK. Treatment of chronic autoimmune urticaria with omalizumab. J Allergy Clin Immunol. 2008;122(3):569-573.

36. Kaplan A, Ledford D, Ashby M, et al. Omalizumab in patients with symptomatic chronic idiopathic/spontaneous urticaria despite standard combination therapy. $J$ Allergy Clin Immunol. 2013;132(1):101-109.

37. Sabroe RA, Fiebiger E, Francis DM, et al. Classification of anti-FcepsilonRI and anti-IgE autoantibodies in chronic idiopathic urticaria and correlation with disease severity. J Allergy Clin Immunol. 2002;110(3):492499.

38. Xu H, Oriss TB, Fei M, et al. Indoleamine 2,3-dioxygenase in lung dendritic cells promotes Th2 responses and allergic inflammation.Proc Natl Acad Sci U S A. 2008;105(18):6690-6695.

39. Pertovaara M, Hasan T, Raitala A, et al. Indoleamine 2,3-dioxygenase activity is increased in patients with systemic lupus erythematosus and predicts disease activation in the sunny season. Clin Exp Immunol. 2007;150(2):274-278.

40. Schroecksnadel K, Winkler C, Duftner C, Wirleitner B, Schirmer M, Fuchs D. Tryptophan degradation increases with stage in patients with rheumatoid arthritis. Clin Rheumatol. 2006;25(3):334-337.

41. McGaha TL, Huang L, Lemos H, et al. Amino acid catabolism: a pivotal regulator of innate and adaptive immunity. Immunol Rev.2012;249(1):135-157.

42. Scott GN, DuHadaway J, Pigott E, et al. The immunoregulatory enzyme IDO paradoxically drives B cell-mediated autoimmunity. J Immunol.2009;182(12):7509-7517.

43. Kiwamoto T, Kawasaki N, Paulson JC, Bochner BS. Siglec-8 as a drugable target to treat eosinophil and mast cell-associated conditions.Pharmacol Ther. 2012;135(3):327-336.

44. Mohamed RW, Fathy A, el-Sayed AE. Increased circulating FcepsilonRII-bearing B-lymphocytes and serum levels of IL-4 in non-autoreactive chronic idiopathic urticaria. Egypt $J$ Immunol.2003;10(2):9-18.

45. Sandham DA, Barker L, Brown L, et al. Discovery of Fevipiprant (NVP-QAW039), a Potent and Selective DP2 Receptor Antagonist for Treatment of Asthma. ACS Med Chem Lett. 2017;8(5):582-586. 